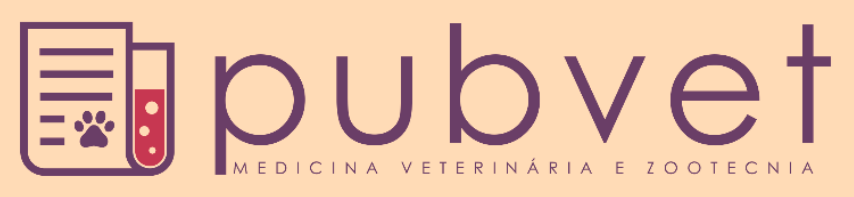

https://doi.org/10.31533/pubvet.v13n5a333.1-8

\title{
Tecnologias de embalagens no pescado: aplicações e tendências
}

\author{
Maria Gabriela Alves $\operatorname{Costa}^{1^{*} \odot}$, Roosevelt de Araújo Sales Júnior ${ }^{1}$, Ayala Oliveira do Vale Souza ${ }^{1}$ \\ ${ }^{I}$ Discente de mestrado, Programa de Pós-Graduação em Produção Animal, Universidade Federal Rural do Semi-Árido, UFERSA, Mossoró, RN, Brasil \\ *Autor para correspondência, E-mail: mgabi_acosta@hotmail.com
}

\begin{abstract}
Resumo. O desenvolvimento de novas tecnologias de embalagens vem crescendo com a finalidade de garantir a qualidade sensorial e nutricional dos alimentos, bem como a segurança alimentar do consumidor, pois evitam a deterioração física, química e microbiológica do alimento. Com isso, o presente trabalho objetivou a busca, teórica, sobre as tecnologias ativas aplicadas em embalagem para a conservação do pescado. Para tanto, foi realizado um levantamento bibliográfico de trabalhos científicos nas bases de dados Science Direct, Periódicos CAPES e Scientific Electronic Library Online utilizando os seguintes descritores: Embalagem, atmosfera modificada pescado, active packaging fish $\mathrm{e}$ intelligent packaging fish.
\end{abstract}

Palavras chave: atmosfera modificada, conservação, embalagem ativa e inteligente

\section{Fish packaging technologies: applications and trends}

Abstract. The development of new packaging technologies has been increasing with the purpose of guaranteeing the sensorial and nutritional quality of the food, as well as the food safety of the consumer, since they avoid the physical, chemical and microbiological deterioration of the food. With this, the present work aimed at the theoretical search on the active technologies applied in packaging for fish conservation. To do so, a bibliographic survey of scientific works was carried out in the databases Science Direct, Periodical CAPES and Scientific Electronic Library Online using the following descriptors: Packaging, modified atmosphere fish, active packaging fish and intelligent packaging fish.

Keywords: modified atmosphere, conservation, active and intelligent packaging

\section{Tecnologías de embalaje en el pescado: aplicaciones y tendencias}

Resumen. El desarrollo de nuevas tecnologías de envases viene creciendo con la finalidad de garantizar la calidad sensorial y nutricional de los alimentos, así como la seguridad alimentaria del consumidor, pues evitan el deterioro físico, químico y microbiológico del alimento. Con ello, el presente trabajo objetivó la búsqueda, teórica, sobre las tecnologías activas aplicadas en embalajes para la conservación del pescado. Para ello, se realizó un levantamiento bibliográfico de trabajos científicos en las bases de datos Science Direct, Periódicos CAPES y Scientific Electronic Library Online utilizando los siguientes descriptores: Embalaje, atmósfera modificada pescado, active packaging fish y intelligent packaging fish.

Palabras clave: atmósfera modificada, conservación, embalaje activo e inteligente

\section{Introdução}

Entre os diversos grupos de alimentos o pescado caracteriza-se como alimentos altamente nutritivos, por serem ricos em micronutrientes, minerais, ácidos graxos essenciais do tipo ômega 3 e ômega-6, de 
fácil digestibilidade e baixo teor de gordura (Santos et al., 2018). Um fator de destaque quando o tema é vida de prateleira, é o alto potencial de deterioração associado principalmente à sua composição química, atividade de água e baixa acidez que favorecem o desenvolvimento de micro-organismos e, consequentemente, mudanças na qualidade da carne que é um fator determinante para a vida útil do produto. Pensando nisso, e que as condições de armazenamento são de suma importância para evitar deterioração do pescado e garantir vida de prateleira, bem como trazer novas tecnologias que possam ser empregadas na conservação do pescado no Brasil de forma que não seja a vácuo, enlatado ou congelado (Pimenta Neto, 2013).

A qualidade dos alimentos é um tema de grande relevância que depende de vários fatores intrínsecos à matéria prima, processamento dos produtos alimentares e/ou armazenamento e para garantir essas características o emprego de tecnologias processuais diversas vem sendo aplicadas. Dentre essas tecnologias as tecnologias aplicadas em embalagens, as quais dizem respeito a um conjunto de medidas, processos e técnicas que associação à ciência de alimentos, o processamento, a preservação e as tecnologias alimentares para garantir sua efetividade vêm sendo bastante pesquisada e empregada nas indústrias e laboratórios (Teixeira \& Garcia, 2016; Tomita et al., 2006; Vieira et al., 2004).

As embalagens desempenham importante papel para a conservação do alimento, o que é fundamental para o consumidor e para a indústria, pois evita a deterioração física, química e microbiológica e, consequentemente, contribui para qualidade e segurança do alimento (Nagarajarao, 2016). O desenvolvimento das embalagens vem desde a origem da humanidade quando se viu a necessidade de transportar e estocar a água, sendo as primeiras embalagens feitas de crânios de animais, chifres ocos e conchas. Com o passar o tempo tem se estudado novos materiais, funções, tamanhos e formas, principalmente devido seu papel fundamental na comercialização dos alimentos (Silva \& Leite, 2010). A embalagem é definida como invólucro, recipiente ou qualquer forma de acondicionamento, removível ou não, destinada a cobrir, empacotar, envasar, proteger ou manter, especificamente ou não, matériasprimas, produtos semielaborados ou produtos acabados (Kerry et al., 2006; Silva \& Leite, 2010). Nos alimentos, atua como barreira entre o alimento e o ambiente, controlando a taxa de transferência de calor, umidade, gases, transmissão de luz e o movimento dos micro-organismos ou insetos (Fang et al., 2017; Polkinghorne et al., 2018). Apesar das funções que as embalagens desempenham serem amplas e variadas, estas muitas vezes é pouco observada e valorizada por alguns consumidores, possivelmente, pela falta de conhecimento acerca da sua importância, o que precisa ser enfatizado. Outro fator que contribui para este aspecto é que no momento em que a maioria dos consumidores entra em contato com a embalagem sua função e/ou interação com o produto já está quase concluída (Gómez-Estaca et al., 2014; Mohan et al., 2016).

Dessa forma, o objetivo do presente trabalho foi trazer um levantamento teórico sobre as tecnologias de embalagens no pescado, incluindo aplicações de tendências.

\section{Embalagens para alimentos}

As embalagens podem apresentar várias definições de acordo com a função que desempenham. Para Realini \& Marcos (2014), a embalagem não se limita apenas as funções de proteger e conservar. Existem outros atributos, como: proteger o alimento contra contaminações ou perdas, facilitar e assegurar o transporte, facilitar a distribuição do alimento, identificar o conteúdo em qualidade e quantidade, identificar o fabricante e o padrão de qualidade, atrair a atenção do comprador, induzir o consumidor para a compra e informar o consumidor sobre composição, valor nutritivo e outras características do alimento, conforme legislação.

A seleção do tipo de embalagem para os variados produtos disponíveis no mercado depende de vários fatores, tais como: o tipo de produto, os requisitos de proteção, a vida útil requerida para o produto, o mercado a que se destina e o circuito de distribuição e venda. Quanto à função das embalagens, elas geralmente são divididas em três níveis: primária, secundária e a terciária. A embalagem primária entra em contato direto com o produto e tem a função de conservar e conter o produto. A embalagem secundária entra em contato com as embalagens primárias e tem finalidade de protegê-las das ações físico-mecânica durante a distribuição. A embalagem terciária, que agrupa diversas embalagens primárias ou secundárias, facilita o transporte dos produtos (Dainelli et al., 2008; Fang et al., 2017; Silva \& Leite, 2010). 
As embalagens de alimentos são estratégias importantes que podem ser decisivas como vantagem competitiva na indústria de alimentos. Portanto, há constante desafio visando atender às exigências dos consumidores, fornecendo embalagens modernas, práticas, que preservem os alimentos e sejam viáveis ambiental e economicamente. As tecnologias que estão surgindo têm a função de prolongar a vida útil do alimento e apresentam papel importantíssimo na preservação e conservação dos produtos, dentre elas temos: embalagem em atmosfera modificada, ativa e inteligente (Nagarajarao, 2016; Seyfert et al., 2004).

\section{Embalagem em atmosfera modificada em pescado: aplicações e tendências}

A Embalagem em Atmosfera Modificada (EAM) se baseia na substituição do ar atmosférico dentro de uma embalagem pela mistura de concentrações fixas de outros gases. Esse processo aplicado ao pescado ocorre de forma ativa, do seguinte modo: o produto é posto dentro da embalagem, em seguida é retirado todo o ar atmosférico criando um vácuo, que também se encaixa como uma atmosfera modificada, e logo após é adicionado uma concentração gasosa pré-selecionada de $\mathrm{O}_{2}, \mathrm{CO}_{2}$ e $\mathrm{N}_{2}$ (Cooksey, 2014; McMillin, 2008; Vergara \& Gallego, 2001). O O $\mathrm{O}_{2}$ está associado a diversas reações bioquímicas em alimentos, em peixes gordos, por exemplo, sua concentração pode intensificar o ranço oxidativo, por outro lado, em peixes magros, tem sido utilizado para reduzir a perda por gotejamento e alterações na cor. Para aumentar a vida de prateleira dos alimentos nas embalagens em atmosfera modificada pela presença de bactérias deteriorativas aeróbicas é necessário o uso de baixos níveis de $\mathrm{O}_{2}$ ou até mesmo sua ausência, pois assim, inibe a ação desses microrganismos (Realini \& Marcos, 2014; Vitale et al., 2014).

$\mathrm{O} \mathrm{CO}_{2}$ é um gás incolor, solúvel em alguns compostos orgânicos e muito importantes na embalagem em atmosfera modificada por conta da sua atividade bacteriostática e fungistáticas. Na realidade, é o gás responsável pela inibição dos microrganismos deteriorantes e sua ação está associado ao $\mathrm{pH}$ pois com a presença do dióxido de carbono o $\mathrm{pH}$ fica ácido e interfere no crescimento de microrganismos deteriorantes aeróbicos. $\mathrm{O} \mathrm{N}_{2}$ é um gás inerte, inodoro, sem gosto e utilizado em embalagem de atmosfera modificada como um enchimento em substituição do $\mathrm{O}_{2}$, pois uma de suas características é de apresentar baixa solubilidade tanto na água quanto na gordura (Mantilla et al., 2010).

$\mathrm{Na}$ tabela 1 temos alguns exemplos de espécies e produtos de pescado, que foram submetidos ao teste de vida de prateleira em atmosfera modificada em uma determinada temperatura de armazenamento. A temperatura é um fator fundamental para essa tecnologia, pois está diretamente associado ao gás $\mathrm{CO}_{2}$, sem um armazenamento adequado (entre $0-4^{\circ} \mathrm{C}$ ) o dióxido de carbono que se encontra dissolvido no produto é perdido, possibilitando que os microrganismos se proliferem e acabem deteriorando o produto. A atividade inibitória do $\mathrm{CO}_{2}$ só acontece em baixas temperaturas justamente, pelo fato, de que a solubilidade do gás em água aumenta com a diminuição da temperatura, devido a isso é tão importante a técnica de embalagem em atmosfera modificada juntamente com o resfriamento (Soccol \& Oetterer, 2003).

Tabela 1. Pesquisas com avaliação de vida útil do pescado e derivados utilizando a tecnologia de embalagem em atmosfera modificada

\begin{tabular}{llcrr}
\hline Espécie do pescado & \multicolumn{1}{c}{ Atmosfera modificada } & $\begin{array}{c}\text { Temperatura } \\
\text { armazenamento }\end{array}$ & $\begin{array}{c}\text { Vida de } \\
\text { prateleira }\end{array}$ & Referências \\
\hline Sardinha (Sardinella brasiliensis) & $100 \% \mathrm{CO}_{2}$ & $2 \pm 2^{\circ} \mathrm{C}$ & 18 dias & Teodoro et al. (2007) \\
Tilápia do Nilo (Oreochromis niloticus) & $40 \% \mathrm{CO}_{2}, 60 \% \mathrm{~N}_{2}$ +irradiação $(1,5 \mathrm{~Gy})$ & $0 \pm 1^{\circ} \mathrm{C}$ & 13 dias & Monteiro et al. (2012) \\
Filé de Atum-rabilho frescos & $100 \% \mathrm{~N}_{2}$ & $3{ }^{\circ} \mathrm{C}$ & 18 dias & Torrieri et al. (2011) \\
Camarão branco do pacífico (L. vannamei) & $75 \% \mathrm{CO}_{2}, 10 \% \mathrm{O}_{2} \mathrm{e} 15 \% \mathrm{~N}_{2}$ & $8^{\circ} \mathrm{C}$ & 11 dias & Wang et al. (2016) \\
Filés de Tilápia-do-Nilo (O. niloticus) & $\mathrm{A}$ vácuo & $4 \pm 1^{\circ} \mathrm{C}$ & 18 dias & Guerra et al. (2017) \\
Caranguejo (Portunus trituberculatus) & $10 \% \mathrm{O}_{2}, 60 \% \mathrm{CO}_{2} \mathrm{e} 30 \% \mathrm{~N}_{2}$ & $-3 \pm 1{ }^{\circ} \mathrm{C}$ & 18 dias & Sun et al. (2017) \\
Lombo de Atum (Thunnus albacares) $)$ & $100 \% \mathrm{CO}_{2}$ & $2 \pm 2^{\circ} \mathrm{C}$ & 0 dias & Souza (2004) \\
\hline
\end{tabular}

\section{Embalagem ativa em pescado: Aplicações e tendências}

A embalagem ativa é uma tecnologia que incorpora e, ou imobiliza certos aditivos à embalagem em vez da incorporação direta no produto. Essa alteração envolve a inclusão de alguns aditivos ao filme, que é feito a embalagem, e tem o objetivo de prolongar a vida de prateleira e manter as características do produto (Vital et al., 2018; Vital et al., 2016). As principais técnicas em embalagens ativas dizem 
respeito a substâncias que absorvem oxigênio, umidade e odor, e aquelas que emitem dióxido de carbono, agentes antimicrobianos, antioxidantes e aromas (Nagarajarao, 2016).

A pesquisa sobre a embalagem ativa em relação às propriedades antimicrobianas e antioxidantes tem crescido com a finalidade de proteger os alimentos contra reações oxidativas e crescimento microbiano. Outra característica que chama atenção é que com isso podem ser embalados produtos naturais bem como aplicado ingredientes naturais, como corantes derivados de fontes naturais e produtos que substituam os aditivos sintéticos. As substâncias naturais, como $\alpha$-tocoferol, óleos essenciais e extratos de plantas têm sido incorporadas em polímeros para a preparação das embalagens (Atarés \& Chiralt, 2016; Fang et al., 2017; Ribeiro-Santos et al., 2017).

Os agentes antimicrobianos podem ser incorporados diretamente à matriz polimérica em rótulos, etiquetas ou estar contidos em sachês. Esses produtos podem ser submetidos às técnicas de incorporação e/ou imobilização (Cooksey, 2014; Ribeiro-Santos et al., 2017). Na primeira, o antimicrobiano é liberado da embalagem ao longo do tempo e, consequentemente, a atividade pode ser estendida durante a distribuição dos alimentos. Por outro lado, na imobilização, a difusão dos compostos para a superfície do alimento ocorre de maneira controlada. Ou seja, estão presentes em menor porção e favorece a comercialização, o que traz uma procura maior do consumidor devido à demanda atual por produtos mais próximos ao natural (Fang et al., 2017; Realini \& Marcos, 2014).

Porém, ainda existem alguns desafios em relação a essa técnica, que são: as características de solubilidade e tamanho dos antimicrobianos, as condições de estocagem e distribuição, método de preparação do filme e a relação do antimicrobiano e o polímero (Fang et al., 2017).

Tabela 2. Pesquisas com avaliação de vida útil de pescado e derivados utilizando a tecnologia de embalagem ativa

\begin{tabular}{|c|c|c|c|c|c|}
\hline Pescado & Embalagem ativa & $\begin{array}{l}\text { Temperatura } \\
\text { armazenamento }\end{array}$ & $\begin{array}{l}\text { Vida de } \\
\text { prateleira }\end{array}$ & Atividade & Referências \\
\hline Peixe barracuda & $\begin{array}{l}\text { Filme ativo a base de quitosana } \\
\text { incorporado com gengibre }\end{array}$ & $2^{\circ} \mathrm{C}$ & 20 dias & Antimicrobiana & Remya et al. (2016) \\
\hline Filé de peixe & $\begin{array}{l}\text { Filme ativo com óleo essencial de } \\
\text { orégano }\end{array}$ & $4^{\circ} \mathrm{C}$ & 10 dias & $\begin{array}{l}\text { Antioxidante e } \\
\text { antimicrobiana }\end{array}$ & Cardoso et al. (2017) \\
\hline Filé de atum-rabilho & $\begin{array}{l}\text { Filmes ativos à base de óleo essencial } \\
\text { de soja-montmorilonita-cravo }\end{array}$ & $2^{\circ} \mathrm{C}$ & 12 dias & $\begin{array}{l}\text { Antioxidante e } \\
\text { antimicrobiana }\end{array}$ & Echeverría et al. (2018) \\
\hline $\begin{array}{l}\text { Camarões brancos do } \\
\text { pacífico }\end{array}$ & $\begin{array}{l}\text { Bicamada em polietileno contendo } \\
\text { óleos essenciais de alecrim e canela }\end{array}$ & $4^{\circ} \mathrm{C}$ & 4 dias & $\begin{array}{l}\text { Antioxidante e } \\
\text { antimicrobiana }\end{array}$ & Dong et al. (2018) \\
\hline
\end{tabular}

\section{Embalagem inteligente em pescado: Aplicações e tendências}

A embalagem inteligente é definida como uma embalagem que monitora as propriedades dos alimentos embalados durante seu transporte e armazenamento. Ou seja, informa ao consumidor, fabricante e varejista sobre as mudanças cinéticas relacionadas com a qualidade dos alimentos (Nagarajarao, 2016). Podem ser compostos por rótulos, etiquetas ou filmes que proporcionam maiores possibilidades de monitoramento da qualidade do alimento acondicionado. Os dispositivos utilizados hoje são: código de barras, etiquetas de identificação de radiofrequência, indicadores de tempotemperatura, indicadores de gás e de patógenos (Ahvenainen, 2003; Han et al., 2005). O código de barras foi introduzido em 1970 e se tornou algo indispensável no mercado para facilitar o controle dos produtos. É um símbolo legível por máquina óptica e apresenta um padrão de barras e espaço com 12 dígitos de dados, onde estão incluídas diversas informações que vai desde data de embalagem do alimento, peso do pacote, lote, informações nutricionais e o endereço do site do fabricante (Soares et al., 2009).

As etiquetas de identificação por radiofrequência (RFID) são mais vantajosas que os códigos de barra, armazenando mais informações e não necessitando de linhas, nem de contato direto com a leitora de código de barras. Porém, essa tecnologia ainda não pode substituir o código de barras porque apresenta alto custo e uma rede de informações mais complexa. As etiquetas de RFID nada mais são que uma tecnologia de armazenamento de dados eletrônicos de produtos que transmitem informações sem fio para a rastreabilidade do pacote com a finalidade de evitar fraudes, melhorar as perdas econômicas, otimizando o transporte, manuseio e condições de armazenamento (Realini \& Marcos, 2014). 
Dentro das embalagens inteligentes ainda temos os indicadores de tempo temperatura (TTI). Esses indicadores são dispositivos simples e baratos que estão presentes nos produtos, desde seu transporte até os pacotes individuais de consumo, com a finalidade de mostrar mudanças na temperatura. A mudança de temperatura nos alimentos pode colocar em risco todo o sistema, pois uma alteração de temperatura pode levar a uma perda da qualidade do produto. Para evitar isso os TTI são frequentemente utilizados como etiquetas em pacotes, em que um material termo sensível imita a taxa de perda de um fator de qualidade para a produção de uma cor ou de cores de contraste para uma escala de referência (Taoukis \& Labuza, 2003; Tsironi et al., 2009). Os tipos principais de sistemas de TTI comercializados apresentam a seguinte classificação: TTI's à base de difusão, onde há a difusão de ésteres coloridos que formam uma cor que contrasta com uma escala de referência, os TTI's enzimáticos, como o próprio nome já sugere, ocorre uma reação enzimática que altera as condições ambientais como o pH, e em seguida, há a mudança de cor e TTI's à base de polímero, isto é, ocorre uma reação de polimerização onde forma um contraste de cor com uma referência escala (Taoukis \& Labuza, 2003).

A qualidade do produto e sua vida de prateleira também apresenta relação com o gás no interior da embalagem. A composição do gás dentro da embalagem muda com o tempo devido à atividade do alimento, da natureza e das condições ambientais. Para isso existem os indicadores de gases, que são utilizados para detectar fugas de gases. Por exemplo, nos sistemas de embalagem em Atmosfera Modificada pode detectar saída indesejada de nitrogênio, dióxido de carbono ou oxigênio (Fang et al., 2017; Yam et al., 2005). Esses indicadores de gases são dispositivos pequenos e se apresentam em forma de etiqueta ou são impressos na embalagem, reagindo à mudança de cor quando há uma alteração na concentração e composição dos gases presentes, o que facilita interpretação por parte do consumidor (Soares et al., 2009).

Além desses indicadores citados acima que são de suma importância para a qualidade e segurança alimentar, há também os biossensores que estão associados à detecção de microrganismos patógenos e toxinas em alimentos. Essa detecção ocorre em tempo real e esse sistema usa elementos biológicos como enzimas, anticorpos, ácidos nucleicos, corantes de $\mathrm{pH}$, entre outros para constar a formação de metabolitos secundários (Poças \& Delgado, 2008).

Por outro lado esse método apresenta desafio por causa da falta de sensibilidade, seletividade, confiabilidade, tempo rápido de resposta e aplicabilidade no rotulo na embalagem do alimento para um melhor aproveitamento dessa tecnologia (Fang et al., 2017).

Tabela 3. Pesquisas com avaliação de vida útil de pescado e derivados utilizando a tecnologia em embalagem inteligente

\begin{tabular}{|c|c|c|c|c|c|}
\hline Pescado & Embalagem Inteligente & Atividade & $\begin{array}{l}\text { Temperatura } \\
\text { Armazenamento }\end{array}$ & $\begin{array}{l}\text { Vida de } \\
\text { Prateleira }\end{array}$ & Referências \\
\hline Peixe & $\begin{array}{l}\text { Tecnologia de etiqueta } \\
\text { de identificação por } \\
\text { radiofrequência (RFID) }\end{array}$ & $\begin{array}{l}\text { Monitoramento da qualidade } \\
\text { do peixe embalado }\end{array}$ & $\begin{array}{l}4^{\circ} \mathrm{C} \\
28^{\circ} \mathrm{C}\end{array}$ & $\begin{array}{l}5 \text { dias } \\
1 \text { dias }\end{array}$ & $\begin{array}{l}\text { Chung et al. } \\
\text { (2017) }\end{array}$ \\
\hline $\begin{array}{l}\text { Fatias de Prionace } \\
\text { glauca e lulas-flecha } \\
\text { Nototodarus sloanii }\end{array}$ & $\begin{array}{l}\text { Tecnologia dos } \\
\text { indicadores de tempo- } \\
\text { temperatura (TTI) - } \\
\text { UV e enzimático }\end{array}$ & $\begin{array}{l}\text { Validar sistema de cadeia de } \\
\text { frio baseado em TTI para } \\
\text { monitorar a vida de prateleira } \\
\text { do pescado congelado }\end{array}$ & $15^{\circ} \mathrm{Ca}-5^{\circ} \mathrm{C}$ & --- & $\begin{array}{l}\text { Tsironi et al. } \\
\text { (2016) }\end{array}$ \\
\hline Truta arco-íris & $\begin{array}{l}\text { Tecnologia de } \\
\text { nanofibras de acetato } \\
\text { de celulose e alizarina }\end{array}$ & $\begin{array}{l}\text { Investigar a capacidade de } \\
\text { nanofibras eletrônicas } \\
\text { sensíveis ao pH em monitorar } \\
\text { a deterioração de peixes }\end{array}$ & $4^{\circ} \mathrm{C}$ & 12 dias & $\begin{array}{l}\text { Aghaei et al. } \\
\text { (2018) }\end{array}$ \\
\hline
\end{tabular}

\section{Considerações finais}

A tecnologia de embalagens é essencial para atender o mercado e a demanda da indústria do pescado, apresentando grandes avanços visando inovação e adaptação às novas exigências tecnológicas e do consumidor. Contudo, é importante enfatizar que estudos ainda são necessários para que as embalagens sejam de confiança, seguras, apresentem características mais próximas do natural do alimento e baratas. Espera-se uma combinação de tecnologias de processamento e de embalagens, como as ativas e as inteligentes, pois são essenciais para uma maior segurança dos alimentos e aumento da vida de prateleira dos produtos de pescado acondicionados. 


\section{Referências bibliográficas}

Aghaei, Z., Emadzadeh, B., Ghorani, B. \& Kadkhodaee, R. (2018). Cellulose acetate nanofibres containing alizarin as a halochromic sensor for the qualitative assessment of rainbow trout fish spoilage. Food and Bioprocess Technology, 111087-1095.

Ahvenainen, R. (2003). Active and intelligent packaging: An introduction. In R. Ahvenainen (Ed.), Novel food packaging techniques (pp. 5-21). Cambridge, U. K. : Woodhead Publishing Series.

Atarés, L. \& Chiralt, A. (2016). Essential oils as additives in biodegradable films and coatings for active food packaging. Trends in Food Science \& Technology, 4851-62.

Cardoso, L. G., Santos, J. C. P., Camilloto, G. P., Miranda, A. L., Druzian, J. I. \& Guimarães, A. G. (2017). Development of active films poly (butylene adipate co-terephthalate)-PBAT incorporated with oregano essential oil and application in fish fillet preservation. Industrial Crops and Products, 108388-397.

Chung, W.-Y., Le, G. T., Tran, T. V. \& Nguyen, N. H. (2017). Novel proximal fish freshness monitoring using batteryless smart sensor tag. Sensors and Actuators B: Chemical, 248910-916.

Cooksey, K. (2014). Chapter 19 - Modified Atmosphere Packaging of Meat, Poultry and Fish. In J. H. Han (Ed.), Innovations in Food Packaging (Second Edition) (pp. 475-493). San Diego: Academic Press.

Dainelli, D., Gontard, N., Spyropoulos, D., Zondervan-van den Beuken, E. \& Tobback, P. (2008). Active and intelligent food packaging: legal aspects and safety concerns. Trends in Food Science \& Technology, 19S103-S112.

Dong, Z., Xu, F., Ahmed, I., Li, Z. \& Lin, H. (2018). Characterization and preservation performance of active polyethylene films containing rosemary and cinnamon essential oils for Pacific white shrimp packaging. Food Control, 9237-46.

Echeverría, I., López-Caballero, M. E., Gómez-Guillén, M. C., Mauri, A. N. \& Montero, M. P. (2018). Active nanocomposite films based on soy proteins-montmorillonite-clove essential oil for the preservation of refrigerated bluefin tuna (Thunnus thynnus) fillets. International Journal of Food Microbiology, 266142-149.

Fang, Z., Zhao, Y., Warner, R. D. \& Johnson, S. K. (2017). Active and intelligent packaging in meat industry. Trends in Food Science \& Technology, 6160-71.

Gómez-Estaca, J., Lopez-de-Dicastillo, C., Hernandez-Munoz, P., Catala, R. \& Gavara, R. (2014). Advances in antioxidant active food packaging. Trends in Food Science \& Technology, 35(1):42-51.

Guerra, N., Maciel, J. F., Araújo, J. \& Cavalheiro, J. M. O. (2017). Efeito da embalagem com atmosfera modificada associada ao ácido ascórbico na vida útil de filés de tilápia-do-nilo (Oreochromis niloticus)/Effect of modified atmosphere packaging associated with ascorbic acid on the shelf life of Nile tilapia fillets (Oreochromis niloticus). Brazilian Journal of Food Technology, 20(e2015045):110.

Han, J. H., Ho, C. H. L. \& Rodrigues, E. T. (2005). Intelligent packaging. In J. H. Han (Ed.), Innovations in food packaging (pp. 138-155). Baltimore: Elsevier Science e Tecnology Books.

Kerry, J. P., O'Grady, M. N. \& Hogan, S. A. (2006). Past, current and potential utilisation of active and intelligent packaging systems for meat and muscle-based products: A review. Meat Science, 74(1):113-130.

Mantilla, S. P. S., Mano, S. B., Vital, H. C. \& Franco, R. M. (2010). Atmosfera modificada na conservação de alimentos. Revista Acadêmica: Ciência Animal, 8(4):437-448.

McMillin, K. W. (2008). Where is MAP going? A review and future potential of modified atmosphere packaging for meat. Meat Science, 80(1):43-65.

Mohan, C. O., Ravishankar, C. N. \& Srinivasagopal, T. K. (2016). Packaging interventions in low temperature preservation of ish-A Review. Food Processing \& Technology, 21-14. doi: doi: 10.15406/mojfpt.2016.02.00026 
Monteiro, M. L. G., Mársico, E. T., Teixeira, C. E., Mano, S. B., Conte Júnior, C. A. \& Vital, H. C. (2012). Validade comercial de filés de Tilápia do Nilo (Oreochromis niloticus) resfriados embalados em atmosfera modificada e irradiados. Ciência Rural, 42(4):737-743.

Nagarajarao, R. C. (2016). Recent advances in processing and packaging of fishery products: A review. Aquatic Procedia, 7201-213.

Pimenta Neto, D. A. (2013). Detecção de adulteração de espécies em pescado e derivados por meio da técnica de DNA Barcoding. Master of Science, Universidade Federal de Minas Gerais, Belo Horizonte.

Poças, F. \& Delgado, T. (2008). Embalagens activas e inteligentes: promessas à vista. Segurança e Qualidade Alimentar, 444-47.

Polkinghorne, R. J., Philpott, J., Perovic, J., Lau, J., Davies, L., Mudannayake, W., . . Thompson, J. M. (2018). The effect of packaging on consumer eating quality of beef. Meat Science, 14259-64.

Realini, C. E. \& Marcos, B. (2014). Active and intelligent packaging systems for a modern society. Meat Science, 98(3):404-419.

Remya, S., Mohan, C. O., Bindu, J., Sivaraman, G. K., Venkateshwarlu, G. \& Ravishankar, C. N. (2016). Effect of chitosan based active packaging film on the keeping quality of chilled stored barracuda fish. Journal of Food Science and Technology, 53(1):685-693.

Ribeiro-Santos, R., Andrade, M., Melo, N. R. \& Sanches-Silva, A. (2017). Use of essential oils in active food packaging: Recent advances and future trends. Trends in Food Science \& Technology, 61132140.

Santos, P. R., Vasconcelos, E. L. Q., Souza, A. F. L., Silva Júnior, J. L. \& Inhamuns, A. J. (2018). Qualidade físico-química e microbiológica de pescado congelado consumido na merenda escolar do estado do Amazonas. PUBVET, 12(5):1-6.

Seyfert, M., Hunt, M. C., Mancini, R. A., Hachmeister, K. A., Kropf, D. H. \& Unruh, J. A. (2004). Accelerated chilling and modified atmosphere packaging affect colour and colour stability of injection-enhanced beef round muscles. Meat Science, 68(2):209-219.

Silva, D. G. \& Leite, V. C. (2010). A importância da embalagem como vantagem logística: um estudo de caso. Tekhne e Logos, 1(3):113-129.

Soares, N. F. F., Silva, W. A., Pires, A. C. S., Camilloto, G. P. \& Silva, P. S. (2009). Novos desenvolvimentos e aplicações em embalagens de alimentos. Ceres, 56(4):370-378.

Soccol, M. C. H. \& Oetterer, M. (2003). Seafood as functional food. Brazilian Archives of Biology and Technology, 46(3):443-454.

Souza, W. G. (2004). Efeito da embalagem em atmosfera modificada sobre conservação de lombo de Atum (Thunnus albacares). Master of Science, Universidade Federal Fluminense, Niterói, Rio de Janeiro, Brasil.

Sun, B., Zhao, Y., Ling, J., Yu, J., Shang, H. \& Liu, Z. (2017). The effects of superchilling with modified atmosphere packaging on the physicochemical properties and shelf life of swimming crab. Journal of Food Science and Technology, 54(7):1809-1817.

Taoukis, P. S. \& Labuza, T. P. (2003). Time-temperature indicators (TTIs). Amsterdan, Holanda: Elsevier.

Teixeira, L. C. \& Garcia, P. P. C. (2016). Qualidade do pescado: captura, conservação e contaminação. Acta de Ciências e Saúde, 1(2):1-15.

Teodoro, A. J., Andrade, E. C. B. \& Mano, S. B. (2007). Avaliação da utilização de embalagem em atmosfera modificada sobre a conservação de sardinhas (Sardinella brasiliensis). Ciência $e$ Tecnologia de Alimentos, 27(1):158-161.

Tomita, R., Furlan, E. F., Neiva, C. R. P. \& Lemos Neto, M. J. (2006). Qualidade físicoquímica do pescado marinho refrigerado em diferentes formas de apresentação. II SIMCOPE-Simpósio de controle do pescado, 1(1):1-4. 
Torrieri, E., Carlino, P. A., Cavella, S., Fogliano, V., Attianese, I., Buonocore, G. G. \& Masi, P. (2011). Effect of modified atmosphere and active packaging on the shelf-life of fresh bluefin tuna fillets. Journal of Food Engineering, 105(3):429-435.

Tsironi, T., Dermesonlouoglou, E., Giannakourou, M. \& Taoukis, P. (2009). Shelf life modelling of frozen shrimp at variable temperature conditions. LWT-Food Science and Technology, 42(2):664671.

Tsironi, T., Giannoglou, M., Platakou, E. \& Taoukis, P. (2016). Evaluation of time temperature integrators for shelf-life monitoring of frozen seafood under real cold chain conditions. Food Packaging and Shelf Life, 1046-53.

Vergara, H. \& Gallego, L. (2001). Effects of gas composition in modified atmosphere packaging on the meat quality of Spanish Manchega lamb. Journal of the Science of Food and Agriculture, 81(14):1353-1357. doi: 10.1002/jsfa.953

Vieira, R. H. S. F., Rodrigues, D. d. P., Barreto, N. S. E., Sousa, O. V., Tôrres, R. C. O., Ribeiro, R. V. \& Madeira, Z. R. (2004). Microbiologia, higiene e qualidade do pescado: teoria e prática. São Paulo, Brasil: Varela.

Vital, A. C. P., Guerrero, A., Kempinski, E. M. B. C., Monteschio, J. O., Sary, C., Ramos, T. R. \& Prado, I. N. (2018). Consumer profile and acceptability of cooked beef steks with edible and active coating containing oregano and rosemary essential oils. Meat Science, 143153-158. doi: https://dx.doi.org/10.1016/j.meatsci.2018.04.035.

Vital, A. C. P., Guerrero, A., Monteschio, J. O., Valero, M. V., Carvalho, C. B., Abreu Filho, B. A. \& Prado, I. N. (2016). Effect of edible and active coating (with rosemary and oregano essential oils) on beef characteristics and consumer acceptability. PlosOne, 1(1):1-15.

Vitale, M., Pérez-Juan, M., Lloret, E., Arnau, J. \& Realini, C. E. (2014). Effect of aging time in vacuum on tenderness, and color and lipid stability of beef from mature cows during display in high oxygen atmosphere package. Meat Science, 96(1):270-277.

Wang, L., Liu, Z., Yang, H., Huang, L. \& Zeng, M. (2016). Optimal modified atmosphere for packaging and its effects on quality and shelf-life of pacific white shrimp (Litopenaeus vannamei) under controlled freezing-point storage at- $0.8^{\circ}$ C. Food Science and Technology Research, 22(2):173183.

Yam, K. L., Takhistov, P. T. \& Miltz, J. (2005). Intelligent packaging: concepts and applications. Journal of food science, 70(1):R1-R10.

Recebido: 9 de abril, 2019.

Aprovado: 15 de maio, 2019.

Publicado: 31 de maio, 2019.

Licenciamento: Este artigo é publicado na modalidade Acesso Aberto sob a licença Creative Commons Atribuição 4.0 (CC-BY 4.0), a qual permite uso irrestrito, distribuição, reprodução em qualquer meio, desde que o autor e a fonte sejam devidamente creditados 